AperTO - Archivio Istituzionale Open Access dell'Università di Torino

\title{
Mona Lisa's Emoji: Digital Civilization and its Discontents
}

\section{This is the author's manuscript}

Original Citation:

\section{Availability:}

This version is available http://hdl.handle.net/2318/1736467

since 2020-04-15T15:47:49Z

Published version:

DOI:10.1080/10350330.2020.1731164

Terms of use:

Open Access

Anyone can freely access the full text of works made available as "Open Access". Works made available under a Creative Commons license can be used according to the terms and conditions of said license. Use of all other works requires consent of the right holder (author or publisher) if not exempted from copyright protection by the applicable law. 


\title{
Mona Lisa's Emoji: Digital Civilization and its Discontents. ${ }^{1}$
}

\author{
Massimo Leone \\ Shanghai University / University of Turin
}

\begin{abstract}
Emojis are presently thus customary in digital communication that they have become part of the communicative habits of most users of social networks. With variations that depend on the particular social network used, on the communicative context, and also on the personality and style of the interlocutors, emojis are, nevertheless, omnipresent. In daily digital conversation, few sentences end without being qualified by a smiling face or other similarly widespread emojis. It is evident to many, included the youngest users of these signs, that representing an attitude of hilarity towards a certain statement by ending its verbal transcription with an emoji 'smiling to tears' is not the same as smiling or laughing to tears when uttering the same content as an oral statement. For reasons that remain to be explained, for instance, most people in digital conversation both smile and cry to tears, as though emotional reactions in this domain were doomed to be conveyed in their most extreme form without the possibility of nuancing them. The major structural difference between an emoji in digital conversation and a facial expression in an oral, face-to-face dialogue, however, comes down to what could be labeled a 'semiotics of the interval'. In mainstream face-to-face conversation, the face is a fundamental interface of social interaction. It can be physiologically controlled in order to guide its communicative effect on the interlocutor; yet, the face is never an entirely semiotic surface. Some of its emotional expressions, such as blushing, for instance, cannot be totally controlled. Moreover, whereas the face must continuously react impromptu to external solicitations, emojis are a typical expression of mastered, differed communication. They are supposed to bestow on digital dialogue an illusion of immediacy, yet they inevitably turn into devices of mediation, resulting from the structural possibility of chiseling a message before sending it out to the interlocutor. The article reflects on this intrinsic difference so as to develop a more general consideration of the cultural reasons and effects of the disappearing of the body from communication and its replacement through digital simulacra.
\end{abstract}

\footnotetext{
${ }^{1}$ Corresponding author:

Massimo Leone: massimo.leone@unito.it
} 
Keywords: Human Communication; Digital Sphere; Body, Embodiment/Disembodiment; Emojis, Semiotics.

"You can only hold a real smile for so long, after that it's just teeth".

(Chuck Palahniuk, Invisible Monsters, 1999, 164)

\section{The gestural common sense.}

The history of human communication could be rewritten considering the extent to which participants expose their bodies in emanating or absorbing meaning (Harrasser 2013; Bateman 2015; RandellMoon and Tippet 2016). A zero degree of such exposition could never be registered, for the human body is, since its very birth, culturally patterned (Barthes 1968; Gontarski 2015; and Bishop, Manghani, and Burgin 2019). The naked human body addressing another naked human body, indeed, is not a purely biological source of meaning manifesting itself in a communicative exchange, but a matrix of exhibitions and occultations composed of gestures, postures, and movements (Duerr 1998; Leone 2010; Leone 2017). The fact itself of modifying the appearance of one's visage through facial expressions can be characterized as an articulation of exposures and withdrawals (Fernández-Dols and Russell. 2017), including the most theatrical of all facial communications: smiling (Trumble 2004). When we humans smile, we uncover our teeth at least partially, or we hint, through the upward movement of our lips, that our teeth might or will be exposed. The evolutionary origin of the smile is still unclear, its explanation hypothetical, but the rationale of this universal facial expression might actually be found in a sort of binary logic: since the mouth can be opened to let the individual nourish itself, it can also be used as one of the most visible elements of facial modification (Aggermann 2013); through no other part of the face, indeed, can a change in the invisible, inner status of the individual be either signaled or simulated in such an economic way. A little experiment can confirm it: let us try to convey the fact that we are extremely happy without moving our mouth from the position that it holds when its muscles are completely relaxed: raising the eyebrows to the utmost extent will not be sufficient, and will be more energetically costly than putting up a simple smile. Also, sharing a complicated code with our interlocutors will be necessary before we can communicate to them through gestures what we are able to express through a simple smile (Feyereisen 2018). The mobility of the mouth, linked to our nature as predators and eaters, has turned the smile into the product of an extremely effective binary code, in which absence and presence are able to express two 
opposite emotional states of relative lack of aggressiveness towards the interlocutor or, on the contrary, the potentiality that such aggressiveness is unleashed.

The biological, evolutionary origin of many facial expressions (Wilkins 2017), among which smiling, should not lead, however, to underestimating the impact that individual exposure to a certain socio-cultural environment exerts on the spontaneous adhesion to local patterns of facial modification in human interaction. A smile is a smile throughout history and geography, yet even purely anecdotic experience as traveler around the world shows that individuals of different societies tend to smile in distinctive ways, by adopting an intensity and degree of facial movements that do not depend only on idiosyncratic manners but reflect, on the contrary, absorption of certain historical and cultural models of emotional expression (Santangelo 2015). Growing up in a certain sociocultural environment, indeed, means also acquiring, both spontaneously and through formal and informal education, the ways in which the members of a community typically control their body in social interaction.

One should not interpret control in a purely restrictive way, though, that is, as an injunction to curtail the bodily expression of one's thoughts, feelings, and emotions, for society controls the body also when it promotes its expressivity rather than its uptightness. As composed Italians know, the sociocultural stereotype that sees the members of the Italian society as particularly keen on expressing themselves through ample and frantic gestures (a stereotype that is exaggerated abroad, for instance, in parodies and imitations, but that rests on the real discrepancy between the Italian gestural culture and those of neighboring societies) leads to the paradoxical situation that, in order to 'look Italian' both in Italy and abroad, these gesturally restrained individuals (including the author of the present essay) are actually compelled to control their bodies in the sense of avoiding a deficiency of gestures rather than an excess of them. A sociocultural standard of bodily manifestation, indeed, exacts a degree of control from the members of a community through imposing either less or more movements that the individual temperament would give rise to, and modeling their shape, energy, and tempo according to patterns that are loosely codified in the gestural common sense of the community but that are, nevertheless, cogent.

\section{Gestural normativity and meta-normativity.}

Such gestural and expressive common sense indicates, for instance, how loudly and blatantly it is appropriate to laugh at someone's wit during a social occasion; the injunction does not usually consist, however, in purely binary alternatives, such as "smiling" versus "not smiling", but in ranges allowing a series of licit reactions between two extremes, in proximity of and, above all, beyond which, gestural behaviors start to appear as abnormal. When someone cracks a joke at a dinner among 
colleagues, for instance, and I do not laugh at all, keeping my body and facial muscles completely still and my voice silent, this total composure might mean that 1) I have not understood the joke, which turns me ipso facto into an outsider in the micro-community and social environment of the dinner; 2) I have understood the joke but I do not like it at all, my gestural reaction, in this case too, marking a sharp contrast between the group and myself; 3) I have understood and do like the joke but I am actually proposing a counter- and meta-joke by forcing myself not to laugh at it. Other nuanced varieties of reactions are also possible, but they all point at the fact that the exposure of the body in public is constantly subject to a diffused normativity, which works sanctioning both excessive and deficient reactions to other people's behaviors in the social environment.

Interpreting such normativity as a simple code, analogous to the formal code of legal normativity, would overlook an essential aspect of its functioning. There are, indeed, occasions in which the diffused and commonsensical social normativity that shapes the body's presence in the environment acts in the same way as a legal code; some gestures and postures are not only social frowned upon but also legally forbidden. In certain sociocultural settings, like present-day Italy or Germany, for instance, the typical salutation gestures of Fascism and Nazism are not only socially stigmatized, but also legally prohibited. Even smiles are sometimes regulated, for instance by the "smile forbidden" signals that one comes across in Cambodia when visiting places connected with the memory of the terrible recent past of the nation under the Khmer Rouges. Also, descending the gradient of cogency in gestural normativity, some particular social occasions, like formal dinners, for example, entail an increased control on the body than informal meals do. Furthermore, some of this control might even be formally codified through verbal description in manner handbooks. Yet, in these circumstances too, gestural normativity would not simply require control on the body but also a meta-control that the analysis of semioticians and ethno-methodologists often neglects.

Understanding the nature, extent, the causes and the effects of such meta-control is, on the contrary, fundamental. Going back to the example of the joke: in order to react to it properly, I must not laugh too little at it; I must not laugh at it too much either. If I do not understand the joke, for instance, but I do not want to give the impression that I have not understood it, or that I have not liked it, I must force myself to laugh, to put on a fake smile and laughter. Yet, unwritten normativity imposes on me not only to smile or laugh, and to do it within the limits of a range of expressive possibilities, but also to do it in a way that does not actually show an intentional adhesion to the gestural code of social normativity. In other words, being a member of a gestural community requires of me that I express and manifest myself according to a standard but with nonchalance. If I fake laughter upon a colleague's joke, I must indeed fake it well, or my failure will be double, both in terms of normativity (I did not understand a joke that everybody understood) and meta-normativity 
(I sought to simulate the effects of my understanding the joke, but I failed). My social environment, therefore, will deduce both that I do not perfectly belong to it and that I badly fake my belonging to it.

\section{Sprezzatura.}

It is important to underline that the kind of meta-normativity that a community imposes on the body of a member and, hence, the meta-control that this meta-normativity entails, are analogous to those which are entailed by the fact of being a native or near-native member of a community of speakers. As a foreigner non-native speaker of a language, I might reach a level at which I completely master its grammar and speak correctly, yet, although I perfectly abide by this first level of normativity, but simultaneously give the impression that I am constantly and painstakingly striving to speak correctly, I would still manifest my condition of non-native speaker. The control that language exacts from me is indeed constantly double: I must speak correctly, and I must also give the impression that such correctness is natural to me, that I do not make any particular effort to abide by it. Italian has a word for this normative nonchalance that is difficult to translate in other languages: "sprezzatura". Designating one of the most central values of Renaissance society and culture, "sprezzatura" labels the ability of those who are able to abide by the complex, often unwritten codes of social behavior and even artistic expression without exuding, for that, any idea of effort or constraint.

As ballet dancers know perhaps better than any other artist, reaching the level at which no impression of effort or unnaturalness in movements is conveyed while dancing, requires a strenuous training, aiming at that prodigious stage where specific habits of movements - which signal their peculiarity exactly by being so distant from the average standards, hence impossible to conform with by common people - are on the contrary executed with such apparent absentmindedness that they appear as perfectly interiorized and absorbed, as fluidly and smoothly mastered as 'simple' walking. The development of an art demands the elaboration of expressive codes that appear exclusive and, therefore, of impervious mastery to the layman, yet it demands also the exertion of a meta-code bestowing on such mastery the impression, or at least the illusion, of absolute ease. It is not only in these elitist and extraordinary domains, however, that a meta-control is required. The "sprezzatura" that the artist or the socialite manifest at the highest degree, indeed, are somehow demanded also from common people, and in general to all those that, in the course of their existence, need to belong to a human group. So as to belong, I must not only behave more or less like the others; I must also give the impression that this behaving more or less like the others does not entail any effort to me.

\section{The cognitive economy of semiosis.}


Understanding the nature of control that communication requires to the members of a community is of essential import for studying the strategies that they adopt, both individually and in groups, so as to cope with the energy that such control and meta-control entail. An evident proportionality obtains between the extent and the speed of change in a sociocultural environment and the cognitive effort that is required to humans living in it so that they may constantly adjust to the normativity and the meta-normativity that rule over social intercourse therein. In the terms of the semiotic philosophy of Charles S. Peirce, habits result from successful interaction with the environment (Kathleen A. Hull and Atkins Richard Kenneth 2017); their utility as crystallized patterns of cognitive, emotional, and pragmatic behaviors consist in their enabling automatic and almost effortless coordination with the other subjects and with the environment itself. Simulating a habit is not having one, exactly insofar a habit should offer a model of interaction that is adopted and lived by as though it had no alternatives. No habit, however, is eternal, exactly for all habits are sociocultural constructs. Indeed, although one might have the impression that speaking one's native language might come to her or him as natural as breathing, it is not exactly like breathing because the latter is a natural disposition selected throughout the evolution of the human species within the environment of the planet earth, whereas the former is a code of social interaction successfully and inadvertently developed by a community over history. Circumstances might arise in which breathing too becomes a non-adaptive pattern of interaction with the environment, for instance in the unlikely circumstance of a planet atmosphere becoming so polluted as to hinder any possibility for humans to intake sufficient oxygen for survival therefrom; in this case too, however, the non-adaptive pattern would not be tantamount to a nonadaptive habit, for the former could not be replaced by an alternative pattern - unless a major genetic mutation occurs in the human species, enabling some individual to breath carbon dioxide instead of oxygen - whereas a habit that proves non-adaptive can be replaced without a change in the biological structure of the individual.

Such opposition, however, should be nuanced. Despite the uneasiness that many semioticians display at the idea of grounding semiotic dynamics into the neurophysiology of the human cognitions, it seems quite evident that habits are not only social constructs, that is, micro-deposits of normativity that members of a community happen to share, but also outcomes of neurological configurations, which ultimately consist in certain synaptic arrangements in the brain. Learning a language, for instance, changes the synaptic configuration of one's brain, to the point that unlearning it ceases to be a voluntary option. The fact that the fixation of interpretive habits, be they in the sphere of cognition, emotion, or action, translates into specific arrangements in the hardware of the mind, that is, in synaptic configurations in the brain, results in their showing a certain degree of inertia in relation to change in the environment. Brains show different degrees of plasticity, yet they all work also as 
mnemonic devices, in which successful interaction with the environment is largely based on shaping, storing, and automatically retrieving patterns of behaviors that have proven successful in the past. The human brain, in other words, allows adaptive interactions with the environment through the formation of habits, and also allows these habits to be modified in relation to changes in the environment, yet at a speed that does not exceed a certain range. Sociocultural conditions and idiosyncratic levels of plasticity can widen this range, yet its measure is nevertheless limited: if the extent of change in the environment exceeds it, or its speed dramatically accelerates, then habits become a hindrance more than a device; in other terms, the crystallization of interpretive patterns through memory turns into a ballast that, nevertheless, cannot be replaced given the neurophysiological nature of the human cognition.

Acquiring a language as a 'mother tongue' means absorbing a series of structured habits of verbal interaction at an age that is characterized by high levels of brain plasticity, so that these habits might turn into a "second nature" in exchanges within one's own linguistic community, to be conducted in a seemingly effortless manner. Migrating into a different linguistic community at an old age, however, might entail the impossibility to acquire analogous habits again, or to acquire them in a way that does not allow their fixation as 'second nature', so giving rise to either imperfect interactions with the new community (lack at the level of linguistic normativity), or to 'artificial' interactions (lack at the level of linguistic meta-normativity).

\section{Semiosis and technology.}

Given this theoretical framework, it is important to underline that the cognitive mechanism diagrammatized by Peirce under the name of semiosis may well be abstractedly universal but is concretely affected by the conditions in which, in the environment, habits are given the possibility to crystallize out of the unceasing dynamic of unlimited semiosis. Indeed, if habits are ways of connecting representamens and objects (i.e., interpretations and reality) that prove successful in pragmatic terms, and mainly in relation to the interactions that such habits enable within a community of interpreters, the most important element to take into account in order to measure the viability of such dynamic in the environment is technology, conceived as the series of operations and devices that allow human beings to change the environment. Being cognitively endowed with the faculty of language, human beings are intrinsically able to change the environment in sophisticated ways, mainly because they are actually capable of imagining and, therefore, planning these changes. Technological development, though, has led to a situation in which human beings have actually given rise to non-natural environments (or to hybrid environments) in which non-human agencies can now alter the environment itself in ways that are not immediately foreseeable or controllable by human 
beings themselves (unless they operate in specific conditions, like computer science analysts, for instance).

The brain of the individual that would linguistically interact with his or her fellows in a medieval village is not physiologically different from the brain of the individual that would linguistically interact with them in an early modern harbor, which is not physiologically different, again, from the brain of the teenager immersed in the augmented reality of a present-day metropolis, yet the amount of change with which these three brains are due to cope is exponentially different. One should wonder, to this regard, whether the amount and speed of change that human technology ends up bringing about in the environment are not such as to actually backfire, turning the brain itself into a non-adaptive device for navigating into the hyper-complexity of the augmented algorithmic world.

\section{Diagrams and schemes.}

From this perspective, several patterns of behaviors and especially technological devices can be read as attempts at coping with a reality in which the amount and speed of change exceed the human capacity for cognitive adaptation. Despite the variety of these behaviors and devices, indeed, they all seem to be underpinned by the same logic, that is, seeking to reduce either the amount or the speed of informational changes from the environment processed by the mind, thus resulting in two trends: on the one hand, the production of various types of diagrams; the main characteristics of changes are summarized in a schematic form that selects invariants, determines the remaining variables, and produces a meta-code that, leaving aside the latter and condensing the former, enables human beings to live by their habitual interpretants also in situations of frantic alterations of the environment. On the other hand, the second trend consists in freezing the environment in a mnemonic representation, which disregards the ensuing variations and bestows a certain stability to the relation between mind and reality.

The first trend underpins the emergence of quantitative representations of the environment and is increasingly fundamental in the digital world: given the amount of information that is produced and circulated in the web second by second, no qualitative heuristics could ensure cognitive survival; algorithms that constantly scan this massive production, summarizing it into a statistic diagram, are fundamental so as to let users reasonably access the mesh of constantly changing information in the digital sphere. The web is continuously altered by micro-variations that are unceasing and multifarious as the waves of the ocean, yet algorithms offer users a relatively stable cartography of it, whose schematizations are, of course, biased by the particular statistical logic adopted in scanning the digital seas, but which nevertheless provide one at least with an illusion of their navigability. 
The second trend undergirds the emergence of qualitative representations of the environment, which are equally fundamental in the digital sphere. In this case, though, they do not stem from a statistic reduction of complexity but from a visual fixation of it, which choses one stage in the frantic evolution of reality and turns it into a static representation. If diagrams and other statistic abstractions are increasingly adopted to summarize the massive and constantly changing amount of information that circulates throughout the digital web, visual representations are more and more adopted so as to fixate subjective reactions to it. In the digital world, statistic diagrams replace reality, whilst visual representations substitute the mind. There is an evident link between, on the one hand, these two strategies of cognitive coping, as well as the kind of behaviors, devices, and patterns that they give rise to, and, on the other hand, two of the three types of signs commonly identified with reference to the semiotics of Charles S. Peirce. The relation of diagrams to the environment, indeed, is in principle indexical, meaning that the former should translate the indexical force of figures that measure the latter; in most cases, however, that is just an illusion, and the realization of it is often at the basis of many users' frustration; indeed, biases introduced by algorithms in sifting the digital world bring about essentially symbolical diagrams, whose relation to the digital environment, and even more to the analogical environment underneath and beyond it, is purely arbitrary, although often coated with an aura of indexicality. Deep down, algorithms do not represent the world, they rather heavily rewrite it for the sake of computability. As regards visual representations, their iconic dimension is even more evident.

\section{Loss of the indexical aura and disintermediation.}

It is fundamental, therefore, to wonder about the status of the indexical force in this world of diagrams and graphics, in which both claim close resemblance to the ground of semiosis but where both often betray their symbolical and iconic nature. Point of departure of such reflection should be the conviction that there is no such a thing as a pure index, meaning by that a sign that is entirely and directly motivated by its ground through an innocent link of cause and effect. That does not rule out, however, that a rhetoric of pure indexicality plays a central role in the history of human cultures. On the one hand, indexes are intrinsically cultural signs, first of all because they are signs, and no simple effects, so that a scheme of cultural interpretation inevitably intervenes in the constitution of a relation between an indexical representamen and its object. On the other hand, human cultures have always considered it important to cultivate the idea that some signs might be pure indexes, that is, that they are directly and immediately motivated by reality. Many cultures, moreover, spend considerable semiotic energies in order to actually empower this rhetoric of indexicality and to maintain the widespread faith in the straightforward link between signs and reality. The reasons behind this thirst 
for indexicality must be traced back throughout the evolutionary history of the relation between language and reality. The idea that the former is in some kind of causal relation with the latter is indeed somehow reassuring, for such relation seems to ground the operability of reality through language.

The more the amount and speed of change in the environment increase, though, mainly as a consequence of technological innovation, the more the two sorts of cognitive coping strategies that have been described above lose their indexical force, since they must neglect more and more idiosyncratic aspects of reality so as to be able to produce a cartography of it. Semiotic investments that are necessary so as to construct a rhetoric of indexicality around these diagrams and schemata become huge as well. Despite these investments, moreover, frustration about their persistent lack of indexical aura starts to seep into the global imagery and produce a whole series of reactions, often encompassed by the literature on the topic under the label of "disintermediation".

From the semiotic point of view, indeed, disintermediation stems from the widespread feeling that the products of the current cognitive coping strategies have completely lost their indexical aura, that is, their power to directly connect reality and representation, ontology and language; that they leave aside too many important details and singularities in their spatial abstractions and temporal fixations; and, above all, that pernicious agencies are at work behind them so as to hide secret interests under an illusory cover of seeming indexicality. Local sources of re-indexicalization, with their power of semiotic re-enchantment, start to pepper throughout the semiosphere at peripheral and marginal locations. It is evident, though, that their purported indexicality is also somehow staged, meaning that it too must resort to a certain degree of spatial abstraction and temporal fixation in coping with the complexity of reality; furthermore, pseudo-indexical representations of this sort might even be socially more pernicious than those that they seek to replace, first for they are actually poorer in articulating and grasping the complexity of the technologically advanced reality and, second, for their indexical aura is an essentially oppositional and negative one, drawing its strength from the contrast with mainstream diagrams and schemes more than from an actually more effective way to represent singularities.

\section{The aura of the face.}

No serious reflection on the dialectics between semiosis, habits, cognitive strategies, indexical aura, and experiments of re-indexicalization can be carried on without reference to the body. The body, as cultural anthropologists and semioticians have demonstrated in relation to various societies throughout history, is itself a device, meaning that its appearance, functioning, and, above all, semiotic capabilities are systematically modified by patterns of cultural articulation. Yet, there is 
perhaps no 'cultural device' with a stronger indexical aura. Such aura is, indeed, partially motivated, for the cultural shaping of the body does not annihilate its biological ground but rather allows its social manifestation. The face, for instance, is a cultural device of expression, used in extremely various ways across societies in time and space, yet its being biologically rooted is a fact, and plays a fundamental role also in the indexical connotation that surrounds this bodily device. A face is the phenomenological membrane that humans present to the world, and that they feel is the closest surface to their interiority (as well as to the mysterious inner principle that moves it, i.e., consciousness) and, at the same time, a malleable screen exposed to the world and to other human beings, ductile at ease (but not indefinitely) so as to show such interiority to the others and to oneself (in the mirror for instance), but also so as to conceal inner thoughts, feelings, and intentions (Skinner Patricia and Cock Emily, eds. 2018). The possibility of lying, at least to a certain extent, and to use, hence, the face as the face of an actor, or as a mask to hide the truthfulness of inner dispositions, alone would suffice to demonstrate that the face is not only biology exposed to the world, but also matrix patterned by society and culture, to the extent that mismatches are also possible between the veracity of one's facial intentions and the actuality of their expressions.

By virtue of this imagined proximity to consciousness, the face is surrounded by a special aura, yet it too is subject to multiple exercises of representation and simulation, since there is probably no object in human nature that has been so extensively depicted and reproduced through countless visual artifacts (Belting 2013). That is true for the pre-digital world, with an immense proliferation of paintings, portraits, and self-portraits, each representing a farther degree in the escalating human need for giving a visual countenance to personal identity (Magli 2016), but it remains true also of the digital world, wherein the face appears as protagonist in social networks, digital creations, immersive experiences, augmented reality, and videogames.

The face itself is an adaptive device that allows humans to manifest or simulate their intentions and seek to interpret the face of others or unmask their forgery. The biological face has been patterned by different cultural matrixes so as to paradoxically enhance both these capabilities: on the one hand, make up has sought to conform the face, and especially the female face, to the desire of other humans, and namely of the males of the species, thus indicating the unbalance in gender power; on the other hand, continuous attempts have been made so as to improve, through physiognomy, for instance, the human ability for reading the truthful inner nature of other human beings through and beyond their faces (Szmidt and Trzeciak 2017). In prehistorical settings, it was probably sufficient for human beings to distinguish the face of the predator from that of the prey, and the face of the enemy from the neutral face, or from the face of the ally. Technology, though, starting from cultivation and agriculture, led to the formation of vast agglomerates of human beings and to the first cities of history, 
where the previous binary patterns of face recognition and reading were not sufficient anymore. A complex array of facial nuances started to emerge in the environment, and an increasing number of potential situations of simulation. More and more, it became important for citizens to prepare their face for the public sphere, and to sharply distinguish between the face that they would display among family members and friends, the one which they would expose to the outer circle of their acquaintances, and the face presented to strangers.

\section{Facial stereotypes and schemes.}

Given the accrued complexity of face interaction in the city, due to technological advancements enabling a greater number of people to coexist and share space, the need for diagrammatic and schematic representations of the face has also arisen. As regards diagrams, they have undertaken to compress the spatial variety of countenances into types, which in history have played and still play a fundamental role in multi-ethnic environments. As a consequence of the number and variety of faces that surround the individual in his or her interaction with the urban setting, characterized by a complexity that exponentially increases in modernity and even more in post-modernity (also as a result of the new easiness by which humans can now travel across the world) citizens cannot cope with such multiplied range of singularities and show a tendency, therefore, to cluster them into types, which inevitably turn into stereotypes as well. Even in present-day cosmopolitan cities, for instance, ethnic stereotypes show a tendency to naturally emerge as an attempt at simplifying the interethnic interaction among citizens; consistent educational efforts are required to replace this evolutionary mechanism with an attention to the singularity of faces beyond their ethnic belonging.

Symmetrically, whereas in post-modern hyper-diverse cities the spatial variety of faces is crystallized into stereotypical and sometimes even racist habits, their temporal variability too is compressed into schemes that freeze the evolution of the individual face throughout time by choosing and 'canonizing' an instant of this evolution. First adopted in medieval Italy, the communitarian habit of identifying members not through their faces, but through static representations of them, has been widely embraced in modernity and even more in post-modernity (Gates 2011). Nowadays, all state communities recognize their insiders through their faces, and impose on outsiders the same kind of recognition, yet images that in national documents or passports should represent the individuality of a face are actually nothing but temporal abstractions, since they freeze the natural and unstoppable evolution of the face into a single moment. Umberto Eco had already noticed that nobody likes her or his picture on the passport or identity card, yet he neglected to investigate the deep historical and cultural reasons for this dislike: as we object the imposition of spatial and ethnic stereotypes on our faces (Bruce 2017), so we also object the imposition of temporal reductions on our visages: the picture 
that should perfectly grasp the singularity of my face within my community or in its and my relations to other groups, actually inevitably presents a past image of myself, the depiction of an obsolete state of my face, whose indexical aura tends to vanish as the time goes by.

\section{Reenchanting the face.}

As it was theoretically exposed above, the loss of indexical aura that results from the creation of devices, patterns, and behaviors of cognitive simplification, be they diagrams (symbolical simplification, like in ethnic stereotypes) or schemes (iconic reduction, like in passport pictures), gives rise to a dissatisfaction that, often, in its turn brings about attempts at re-indexicalization, attempts whose social by-products the sociological literature generically characterizes as "disintermediation". The face too, which apparently is the most indexical device of human interaction, is subject to this dynamic. As I have sought to demonstrate in other essays, contemporary critical thinking and literature have often emphasized the post-modern tendency to create increasingly isolating and isolated semiotic spheres (Leone Massimo. Forthcoming), a movement through which the semiosphere, theretofore coinciding with the community, has been fragmented into increasingly smaller fractals, each of them becoming a semiosphere in itself, until a sort of bubbly configuration has arisen, wherein each body is socially transmogrified into a private cultural sphere, uncommunicated with its immediate surroundings.

This characterization of the post-modern individual as spatially isolated from the others, though, fails to explain the enormous and spasmodic extent to which individuals nowadays actually seek contact with others, strive for egressing their bubbles, and are prey to a whole series of nostalgic trends concerning 'the era of the community', trends that are often hijacked and capitalized by successful initiatives in the market of goods and political ideas.

Such apparent contradiction, which underlines the progressive self-seclusion and even selfsegregation of the post-modern individual but fails to explain his or her enormous appetite for connection, emerges from overemphasizing the importance of the spatial coordinate to the detriment of the temporal one. Post-modern individuals seek, indeed, to react to the alienating consequences of diagrammatization and schematization of their singularities, yet they do so by adding to the spatial segregation in which they are immersed a temporal confinement. That gives rise to a widespread semiotic syndrome that I have labeled elsewhere as the "civilization of the interval". In it, individuals do not interact with others through the interface of their visage, but through a pre-arranged simulation of it.

Let us resume the passages that have led to this paradoxical strategy of re-indexicalization: technology gives rise to enormous conglomerates of human beings that now happen to share the same 
hyper-complex and hyper-diverse metropolitan space and time; ancestral patterns of human interaction, based on the capacity of presenting one's face and reading the face of others, cannot cope with the exponential variety of stimuli; spatial diagrams and temporal schemes intervene, creating clusters of human faces that abstract their main features to the detriment of faithfulness to singularities in space and time; the lack of aura affecting these abstractions, though, leads to attempts at overcoming the self-segregation that individuals suffer when cased and caged into these spatial and temporal stereotypes; turned into facial abstractions, human beings still feel the insatiable urge to connect to other human beings beyond these stereotypes, they want their singularity to be recognized as distinctive. Everybody in the post-modern world is led to resemble a stereotype yet everybody wants to be unique.

\section{A paradoxical thirst for uniqueness.}

Such thirst for uniqueness - a long-term product of modernity that is increasingly titillated by the market - is unquenchable, especially if it is accompanied by the equally utopian need for such uniqueness to be universally liked. We want to be an unanimously loved singularity. On the one hand, the love that would circulate through a community, the one uniting a national group, for instance, is not sufficient anymore: I do not want to be loved as the member of a group, but as an individual emerging from it, or even towering over it; on the other hand, the singular love that one would previously find in intimate relations, such as the life-long devotion of a husband towards his wife or vice versa, also falls short of satisfying the post-modern individual thirst for universal recognition. Segregated into spatial diagrammatic stereotypes and temporal schematic icons, pushed by modernity and its markets to be, on the contrary, unique, and encouraged to cultivate a desire for the universal recognition of this impossible uniqueness, the post-modern individual seeks to re-enchant himself or herself, to bestow an indexical aura of faithful singularity to the presentations and representations of his or her face in the public sphere, to dis-intermediate the interaction with other human beings. As a consequence, immediacy is sold as a new form of aura.

Whereas in pre-modern times the face would be shown to a restricted circle of individuals, mainly belonging to one's family or village, and whereas in modern times such exposure widened its scope, so giving rise to the strategies of cognitive coping that we have described thus far, in postmodernity the face is shown potentially to the entire world (Zilio 2018). That is the paradoxical, impossible, and ultimately frustrating condition of the post-modern individual: I want to be endowed with a unique face, yet I must show it to a universal audience of strangers; moreover, my satisfaction will depend on the unanimous appreciation by which this audience will receive my face. In the past, no face would be subject to such aesthetic ordeal. Only the face of the king, of the queen, of the pope, 
would simultaneously circulate through everybody's hands, through effigies on coins, for instance, demanding not only the appreciation, but also the devotion and love of all the subjects, to the point that such aesthetic and affective response was codified and even turned into a normative standard: disrespecting the effigy of the souverain was - and in certain human groups still is - a crime.

With the advent of mass communication, many royal and enchanting facial images have started to circulate in the semiosphere, yet they have been in competition with each other. Singers, actors, artists would compete with each other for the privilege of being enthroned by the universal audience, and they would often succumb to the inane effort, disrupting their lives. Nowadays, that selfdestructing frustration that in the remote past would be spared to kings and queens and popes since a rigid norm would immediately eliminate all competitors - and that, in more recent times, has plagued the lives of those who, like Hollywood actors or pop singers, would seek at the same time a feeling of uniqueness and the universal appreciation of an increasingly and more and more terrifying universal audience, is now empoisoning the life of every individual in the post-modern and technological advanced planet. Everybody wants to be a king, but everybody is surrounded by millions of individuals with the same ambition. A gigantic collective neurosis ensues from the conundrum of singularity in the digital world. Economic empires are built through monetizing the unquenchable insecurity of the post-modern individual.

\section{The market of indexicality.}

They sell, first, an illusion of immediacy, proposed as an antidote to the depersonalizing both spatial and temporal abstractions in which the individual is systemically constrained in post-modern societies. If I venture with my physical face into the post-modern communicative arena of the hypercomplex and hyper-diverse metropolis, I shall be read through countless stereotypes or, worse, I shall be ignored. I shall be able to distinctively realize the extent to which other people ignore me, not only in foreign cities, but in my own city, in my own neighborhood, even in my own building or condo elevator. Simultaneously, I will ignore others in the same way. In many circumstances, moreover, this feeling of isolation will turn into one of fear: the city will become, in the socially shared imaginary, not only a place of utmost indifference, but also one in which, if someone pays actual attention to me, it is likely because of his or her harmful intentions. In some present-day cities it is actually becoming impossible to stop another citizen and talk to him or her, even only so as to ask for information, without stirring a feeling of unease and even terror (Leone 2009).

In the digital world, on the contrary, I am sold the illusion that I can constantly maintain control over the appearance of my face, and that I can change its features beyond any imposed diagrammatic or schematic abstraction. In the digital sphere, not only can I replace the icon of my face at will, but 
I can also increasingly modify it through more and more sophisticated devices. Whereas in the past the imperative of maquillage would point to the gender subjection of women to the judgment of the male gaze (McHugh 2013), or to that of the male gaze interiorized by women, now the imperative of digital maquillage affects all those who throw themselves in the chaotic arena of social networks. Frustrated in its confinement, relegated to the symbolical aridness of diagrams or to the iconic imprecision of schemes, the post-modern individual revels into self-aggrandizing delusions of uniqueness and likability, where the anonymity or, worse, the defenselessness of the face in the public sphere is replaced by a dream of immediate recognition, of production and, increasingly, postproduction of simulacra that constantly re-enchant the presentation and re-presentation of the face, keep them in line with the subtlest nuances of the inner self, and, above all, are constantly changeable and monitorable in relation to the quantitative feedback that digital social networks and their algorithms unceasingly provide. My face, as a result, never really ventures into the public, or continues to traverse it in its fragile anonymity, yet its digital version is empowered by new genres, like the selfie, by new formats, like the gif or the meme, by new special effects that were hitherto an exclusive resource of Hollywood actors and actresses and are now offered to everyone for a modest subscription or even for free, if one accepts to be scanned and sold as matrix of possible desires to the market.

That is, in a nutshell, the civilization of the semiotic interval: I seek to escape the confinement of the stereotypes and anonymizations to which the post-modern metropolis condemns me, yet I fall prey to a market that sells me delusions of temporal control as antidote to the impossibility of spatial control, and offers me to connect with other individuals never through my physical face, but through simulacrums of it that, although I can immediately alter and monitor them, are actually never immediate. The ultimate outcome of this market is the creation of a digital zoo from which I shall never be able to escape, for therein my ability to cope with the temporal immediacy of the world, and the insertion of my own physical face into it, atrophies. Analogic make up will not suffice anymore once that I have become accustomed to the universal likability of increasingly sophisticated digital filters. How can I venture into the world, indeed, with a face that I cannot completely change, completely control, completely modify in relation to circumstances, and whose likability, moreover, constantly escapes any precise appraisal? How dreadful it is, coming across in the real world someone whom I have become accustomed to see in digital social networks, and how embarrassing to show oneself to someone who has befriended the beautified version of ourselves! The only way to avoid the disillusion of reality in times of digital solipsism is to remain within the trenches of the interval.

\section{Conclusion.}


In the smile of Mona Lisa, Leonardo miraculously captured the unseizable ambiguity of the human face, of the female countenance, of the young smile, of this almost liquid matrix of flesh and bones and skin that emerges from the canvas together with a culture and a language, which both recognize and denominate therein, in the canvas, two eyes, a nose, a mouth, two lips, and yet must yield to the power of the icon, which in this case, though, is not a diagram stereotypically subsuming a visage into the ideal face of a society or community or group, and it is not either a scheme sacrificing the temporal evolution of the visage, its being tragically subject to the passing of time, but a supremely singular face, for the smile of Mona Lisa, as a result of a prodigious mastery of the pictorial elements and the illusion that they provoke in the gaze, seems to impalpably move, to change as we spectators alter our position in looking at it, but also, more profoundly, as we change in time, as we also proceed inexorably toward death, so that this volatile smile seems to almost accompany us in our unceasing alteration, and mirror the fragile singularity of our uniqueness, with the result that in every instant of our contemplation we might, if our gaze is attentive enough, unceasingly relive the creation and recreation of an aura, an enchantment that flows through and around those sublime lips, since their indexical force was never exhausted in the fixation of a form, and its position, and its colors, but rendered in a paradoxically fluid manner, as if the canvas were able to defy and overcome the paucity of its two dimensions, and even the three dimensions of our own body, to immerse ourselves and itself into a fourth dimension of temporal becoming, where at least we recognize that that smile is not only unique in itself, but also unique in relation to itself, unique at every instant, and different from the previous moment, and from the following one, so emanating towards our awareness the idea of a uniqueness that never turns into stereotype but bestows on this conjunction of smile and gaze the aura of love, which is nothing by the ability to see the uniqueness of the smile of the other along the tragic line that approaches it more and more to eternal fixation, and death.

This is not an emoji.

\section{Disclosure statement}

No potential conflict of interest was reported by the author.

\section{Funding}

This project has received funding from the European Research Council (ERC) under the European Union's Horizon 2020 research and innovation programme (grant agreement No 819649 - FACETS). 


\section{Note on contributor}

Massimo Leone is Tenured Full Professor ("Professore Ordinario") of Philosophy of Communication, Cultural Semiotics, and Visual Semiotics at the Department of Philosophy and Educational Sciences, University of Turin, Italy, Vice-Director for research at the same University, and part-time Professor of Semiotics in the Department of Chinese Language and Literature, University of Shanghai, China. He has been visiting professor at several universities in the five continents. He has single-authored twelve books, edited more than thirty collective volumes, and published more than five hundred articles in semiotics, religious studies, and visual studies. He is the chief editor of Lexia, the Semiotic Journal of the Center for Interdisciplinary Research on Communication, University of Torino, Italy (SCOPUS). He is the winner of a 2018 ERC Consolidator Grant, the most prestigious research grant in Europe.

\section{ORCID: $\underline{\text { https://orcid.org/0000-0002-8144-4337 }}$}

\section{Bibliography.}

Aggermann, Lorenz. 2013. Der offene Mund: Über ein zentrales Phänomen des Pathischen. Berlin: Theater der Zeit.

Barthes, Roland. 1968. Le degré zéro de l'écriture. Suivi de : Eléments de sémiologie. [Bibliotheque Médiations: 40]. Paris: Gonthier.

Bateman, Simone. 2015. Inquiring into human enhancement: interdisciplinary and international perspectives. Health, technology, and society. Houndsmills, Basingsoke; New York, NY: Palgrave Macmillan.

Belting, Hans. 2013. Faces: Eine Geschichte des Gesichts. Munich: C.H. Beck.

Bishop, Ryan, Sunil Manghani, and Victor Burgin. 2019. Seeing degree zero: Barthes/Burgin and political aesthetics. Edinburgh, UK: Edinburgh University Press.

Boneschi, Marta. 2018. Il comune senso del pudore [Intersezioni: 506]. Bologna: Il Mulino.

Bruce, Vicki. 2017. Recognising faces. New York, NY: Routledge.

Duerr, Hans Peter. 1998. Nudité et pudeur: le mythe du processus de civilisation. French trans. from German of Mythos vom Zivilisationsprozess. Bd. 1. Nacktheit und Scham, by Véronique Bodin with the participation of Jacqueline Pincemin; preface par André Burguière. Paris: Maison des sciences de l'homme.

Fernández-Dols, José-Miguel and James A. Russell. 2017. The science of facial expression. New York, NY: Oxford University Press. 
Feyereisen, Pierre. 2018. The cognitive psychology of speech-related gesture. Abingdon, Oxon; New York, NY: Routledge.

Gates, Kelly A. 2011. Our biometric future: facial recognition technology and the culture of surveillance. New York, NY: New York University Press.

Gontarski, S.E. 2015. Creative involution: Bergson, Beckett, Deleuze. Edinburgh, UK: Edinburgh University Press, 2015.

Harrasser, Karin. 2013. Körper 2.0: Über die Technische Erweiterbarkeit des Menschen. [Xtexte]. Bielefeld: Transcript.

Kathleen A. Hull and Atkins, Richard Kenneth. 2017. Peirce on perception and reasoning: From icons to logic [Routledge Studies in American Philosophy]. London, UK and New York, NY: Routledge.

Leone, Massimo. 2009. Virtual Cities and Civic Virtues: The Semiotics of Space in Gated Communities. In Actual and virtual cities (intertextuality and intermediality), ed. Asunción López-Varela Azcárate and Mariana Net, 67-87. Bucharest: Univers Enciclopedic Press.

Leone, Massimo. 2010. Pudibondi e spudorati: Riflessioni semiotiche sul linguaggio del corpo (s)vestito, Rivista Italiana di Filosofia del Linguaggio, 2: 74-94.

Leone, Massimo. 2017. Homo velans: paradossi del velo nella semiosfera contemporanea. In Le verità del velo, 27-45. Ed. Marianna Ferrara, Alessandra Saggioro, and Giusi Viscardi, eds. 2015. Florence: Società Editrice Fiorentina [series “Alti Studi di Storia intellettuale e delle Religioni"].

Leone, Massimo. Forthcoming. El giro digital en la semiótica de las culturas. In Ciberculturas, monographic issue of DeSignis, journal of the Latin American Federation of Semiotics, ed. Eliseo R. Colón Zayas, forthcoming.

Lunceford, Brett. 2018. Public nudity and the rhetoric of the body. Lanham, MD: Lexington Books. Magli, Patrizia. 2016. Il volto raccontato: ritratto e autoritratto in letteratura [Minima: 135]. Milan: Raffaello Cortina editore.

McHugh, Tony. 2013. Faces inside and outside the clinic: A Foucauldian perspective on cosmetic facial modification. Farnham, Surrey, UK: Ashgate Publishing Company.

Randell-Moon, Holly, and Ryan Tippet. 2016. Security, race, biopower: Essays on technology and corporeality. London: Palgrave Macmillan UK.

Restuccia, Frances. 2019. Agamben's political ontology of nudity in literature and art: literary criticism and cultural theory. New York, NY: Routledge.

Santangelo, Paolo. 2015. Laughing in chinese. Rome: Aracne. 
Skinner, Patricia and Cock, Emily, eds. 2018. Approaching facial difference: past and present. London, UK and New York, NY: Bloomsbury Academic.

Szmidt, Olga and Trzeciak, Katarzyna. 2017. Face in trouble: from physiognomics to Facebook. Frankfurt A.M.: Peter Lang GmbH, Internationaler Verlag Der Wissenschaften.

Trumble, Angus. 2004. Brief history of the smile. New York, NY: Basic Books.

Wilkins, Adam S. 2017. Making faces: the evolutionary origins of the human face. Cambridge, MA: The Belknap Press of Harvard University Press.

Zilio, Marion. 2018. Faceworld: le visage au XXIe siècle. Paris: PUF. 